PROCEEDINGS OF THE

AMERICAN MATHEMATICAL SOCIETY

Volume 138, Number 10, October 2010, Pages 3707-3716

S 0002-9939(2010)10504-X

Article electronically published on May 27, 2010

\title{
PROPER ACTIONS ON TOPOLOGICAL GROUPS: APPLICATIONS TO QUOTIENT SPACES
}

\author{
SERGEY A. ANTONYAN
}

(Communicated by Alexander N. Dranishnikov)

\begin{abstract}
Let $X$ be a Hausdorff topological group and $G$ a locally compact subgroup of $X$. We show that the natural action of $G$ on $X$ is proper in the sense of R. Palais. This is applied to prove that there exists a closed set $F \subset X$ such that $F G=X$ and the restriction of the quotient projection $X \rightarrow X / G$ to $F$ is a perfect map $F \rightarrow X / G$. This is a key result to prove that many topological properties (among them, paracompactness and normality) are transferred from $X$ to $X / G$, and some others are transferred from $X / G$ to $X$. Yet another application leads to the inequality $\operatorname{dim} X \leq \operatorname{dim} X / G+\operatorname{dim} G$ for every paracompact topological group $X$ and a locally compact subgroup $G$ of $X$ having a compact group of connected components.
\end{abstract}

\section{INTRODUCTION}

By a $G$-space we mean a completely regular Hausdorff space together with a fixed continuous action of a Hausdorff topological group $G$ on it.

The notion of a proper $G$-space was introduced in 1961 by R. Palais 23] with the purpose of extending a substantial portion of the theory of compact Lie group actions to the case of noncompact ones.

A $G$-space $X$ is called proper (in the sense of Palais [23, Definition 1.2.2]) if each point of $X$ has a, so-called, small neighborhood, i.e., a neighborhood $V$ such that for every point of $X$ there is a neighborhood $U$ with the property that the set $\langle U, V\rangle=\{g \in G \mid g U \cap V \neq \emptyset\}$ has compact closure in $G$.

Clearly, if $G$ is compact, then every $G$-space is proper.

Many important problems in the theory of proper actions are conjugated (see [18, [1], 2], 8], 7]) to the following major open problem:

Conjecture 1. Let $G$ be a locally compact group. Then the orbit space $X / G$ of any paracompact proper $G$-space $X$ is paracompact.

This conjecture is open even if $X$ is metrizable; in this case it is equivalent (see [8]) to the following old problem going back to R. Palais [23]:

Conjecture 2. Let $G$ be a locally compact group and $X$ a metrizable proper $G$ space. Then the topology of $X$ is metrizable by a $G$-invariant metric.

Received by the editors May 15, 2009.

2010 Mathematics Subject Classification. Primary 22A05, 22F05, 54H11, 54H15, 54F45.

Key words and phrases. Proper $G$-space, orbit space, locally compact group, dimension.

The author was supported in part by grants \#IN102608 from PAPIIT (UNAM) and \#79536 from CONACYT (Mexico). 
Due to Palais 23, it is known that Conjecture2 is true for a separable metrizable proper $G$-space $X$ provided the acting group $G$ is Lie. Other special cases are discussed in [20] and [8]. In particular, in [8], Palais' result is extended to the case of a locally compact separable $G$ and a metrizable locally separable $X$.

In this paper we prove Conjecture 1 in an important special case, namely, when $X$ is a topological group endowed with the natural action of its locally compact subgroup $G$ (see Corollary [1.5). We first show that $X$ is a proper $G$-space and then we establish a more general result (Theorem 1.2) which has many interesting applications in the theory of topological groups.

Below all topological groups are assumed to satisfy the Hausdorff separation axiom.

Theorem 1.1. Let $X$ be a topological group and $G$ a locally compact subgroup of $X$. Then the action of $G$ on $X$ given by the formula $g * x=x g^{-1}, g \in G, x \in X$, is proper.

Recall that a subset $S$ of a proper $G$-space $X$ is called $G$-fundamental, or just fundamental, if $S$ is a small set and the saturation $G(S)=\{g s \mid g \in G, s \in S\}$ coincides with $X$.

Here is the key result of the paper:

Theorem 1.2. Let $X$ be a topological group and $G$ a locally compact subgroup of $X$. Then there exists a closed $G$-fundamental set in $X$.

It is easy to prove (see [2, Proposition 1.4]) that in every proper $G$-space $X$, the restriction of the orbit map $p: X \rightarrow X / G$ to any closed small set is perfect (i.e., is closed and has compact fibers). In combination with Theorem 1.2 this yields the following:

Corollary 1.3. Let $X$ be a topological group, $G$ a locally compact subgroup of $X$, and $X / G$ the quotient space of all right cosets $x G=\{x g \mid g \in G\}, x \in X$. Then there exists a closed subset $F \subset X$ such that the restriction $\left.p\right|_{F}: F \rightarrow X / G$ is a perfect surjective map.

This fact has the following immediate corollary about transfer of properties from $X$ to $X / G$ :

Corollary 1.4. Let $\mathcal{P}$ be a topological property stable under perfect maps and also inherited by closed subsets. Assume that $X$ is a topological group with the property $\mathcal{P}$ and let $G$ be a locally compact subgroup of $X$. Then the quotient space $X / G$ also has the property $\mathcal{P}$.

Among such properties $\mathcal{P}$ we single out just some of those which provide new results in Corollary 1.4 these are: paracompactness, countable paracompactness, weak paracompactness, normality, perfect normality, Čech-completeness, being a $k$ space (see [16, $\S 5.1, \S 5.2, \S 5.3, \S 1.5, \S 3.9, \S 3.3]$ ) and stratifiability (see [14]). Thus, we get the following positive solution of Conjecture 1 in an important special case:

Corollary 1.5. Let $X$ be a paracompact topological group and $G$ a locally compact subgroup of $X$. Then the quotient space $X / G$ is paracompact.

In this connection it is in order to recall the following remarkable result of A. V. Arhangel'skii [9]: every topological group is the quotient of a paracompact 
zero-dimensional group. Hence, the local compactness of $G$ is essential in Corollary 1.5 .

We recall that a locally compact group is called almost connected if its space of connected components endowed with the quotient topology is compact.

Corollary 1.6. Let $\mathcal{P}$ be a topological property stable under open perfect maps and also inherited by closed subsets. Assume that $X$ is a paracompact group with the property $\mathcal{P}$ and let $G$ be an almost connected subgroup of $X$. Then the quotient space $X / G$ also has the property $\mathcal{P}$.

Among properties stable under open perfect maps and also inherited by closed subsets we highlight strong paracompactness and realcompactness (see 16, Exercises 5.3.C(c), $5.3 \mathrm{H}(\mathrm{d})$, and Theorem 3.11.4 and Exercises 3.11.G]). Thus, we get the following:

Corollary 1.7. Let $X$ be a strongly paracompact (resp., paracompact and realcompact) topological group and let $G$ be an almost connected subgroup of $X$. Then the quotient space $X / G$ is strongly paracompact (resp., paracompact and realcompact).

Remark 1.8. We note that the converse of the first statement in this corollary is not true. Namely, it is known that the Baire space $B\left(\aleph_{1}\right)$ of weight $\aleph_{1}$ (which is, in fact, homeomorphic to a commutative metrizable topological group) is strongly paracompact and its product with the additive group $\mathbb{R}$ of the reals is not (see [16, Exercises $5.3 \mathrm{~F}(\mathrm{a})$ and $5.3 \mathrm{~F}(\mathrm{~b})])$. Hence, the direct product $X=\mathbb{R} \times B\left(\aleph_{1}\right)$ and its subgroup $G=\mathbb{R} \times\{0\}$ provide the desired counterexample, answering negatively a question from [11. Furthermore, [12, Open Problem 3.2.1, p. 151] asks whether every locally strongly paracompact group is strongly paracompact? The same group $\mathbb{R} \times B\left(\aleph_{1}\right)$ provides a negative answer to this question too. Indeed, since the product of a compact space and a strongly paracompact space is strongly paracompact (see [16, Exercise $5.3 \mathrm{H}(\mathrm{a})])$, we infer that $\mathbb{R} \times B\left(\aleph_{1}\right)$ is locally strongly paracompact.

Combining our Corollary 1.5 with a result of Abels [1, Main Theorem], we obtain the following:

Corollary 1.9. Let $X$ be a paracompact group, $G$ an almost connected subgroup of $X$, and $K$ a maximal compact subgroup of $G$. Then there exists a $K$-invariant subset $S \subset X$ such that $X$ is $K$-homeomorphic to the product $G / K \times S$. In particular, $X$ is homeomorphic to $\mathbb{R}^{n} \times S$ for some $n \geq 1$.

In [10] A. V. Arhangel'skii has studied properties which are transferred in the opposite direction, i.e., from $X / G$ to $X$. The next corollary is a unified result of this sort which implies many of those in [10] as well as provides some new ones.

Corollary 1.10. Let $\mathcal{P}$ be a topological property invariant and inverse invariant of perfect maps, and also stable under multiplication by a locally compact group. Assume that $X$ is a topological group and let $G$ be a locally compact subgroup of $X$ such that the quotient space $X / G$ has the property $\mathcal{P}$. Then the group $X$ also has the property $\mathcal{P}$.

Among such properties we mention just some: paracompactness, being a $k$ space, Čech-completeness (see [16, $\S 5.1, \S 3.3, \S 3.9]$ ). That paracompactness is stable under multiplication by a locally compact group follows from a result of Morita 21 . since every locally compact group is paracompact (even, strongly paracompact [12, Theorem 3.1.1]). 
Corollary 1.11. Let $\mathcal{P}$ be a topological property invariant and inverse invariant of open perfect maps, and also stable under multiplication by a locally compact group. Assume that $X$ is a paracompact group and let $G$ be an almost connected subgroup of $X$ such that the quotient space $X / G$ has the property $\mathcal{P}$. Then the group $X$ also has the property $\mathcal{P}$.

Among such properties we highlight realcompactness (see [16, Theorem 3.11.14 and Exercise 3.11.G], and we also take into account that every locally compact group is realcompact).

Corollary 1.5 is further applied to prove the following Hurewicz type formula:

Theorem 1.12. Let $X$ be a paracompact topological group and $G$ an almost connected subgroup of $X$. Then

$$
\operatorname{dim} X \leq \operatorname{dim} X / G+\operatorname{dim} G .
$$

Remark 1.13 (24]). If in this theorem $X$ is a locally compact group, then, in fact, the equality holds:

$$
\operatorname{dim} X=\operatorname{dim} X / G+\operatorname{dim} G .
$$

All the proofs are given in section 3 .

\section{Preliminaries}

Throughout the paper, unless otherwise stated, by a group we shall mean a topological group $G$ satisfying the Hausdorff separation axiom; by $e$ we shall denote the unity of $G$.

All topological spaces are assumed to be Tychonoff (= completely regular and Hausdorff). The basic ideas and facts of the theory of $G$-spaces or topological transformation groups can be found in G. Bredon [15] and in R. Palais [22. Our basic reference on proper group actions is Palais' article [23]. Other good sources are 20, 1] and 2].

For the convenience of the reader we recall, however, some more special definitions and facts below.

By a $G$-space we mean a topological space $X$ together with a fixed continuous action $G \times X \rightarrow X$ of a topological group $G$ on $X$. By $g x$ we shall denote the image of the pair $(g, x) \in G \times X$ under the action.

If $Y$ is another $G$-space, a continuous map $f: X \rightarrow Y$ is called a $G$-map or an equivariant map if $f(g x)=g f(x)$ for every $x \in X$ and $g \in G$.

If $X$ is a $G$-space, then for a subset $S \subset X$ and for a subgroup $H \subset G$, the $H$-hull (or $H$-saturation) of $S$ is defined as follows: $H(S)=\{h s \mid h \in H, s \in S\}$. If $S$ is the one-point set $\{x\}$, then the $G$-hull $G(\{x\})$ is usually denoted by $G(x)$ and called the orbit of $x$. The orbit space $X / G$ is always considered in its quotient topology.

A subset $S \subset X$ is called $H$-invariant if it coincides with its $H$-hull, i.e., $S=$ $H(S)$. By an invariant set we shall mean a $G$-invariant set.

For any $x \in X$, the subgroup $G_{x}=\{g \in G \mid g x=x\}$ is called the stabilizer (or stationary subgroup) at $x$.

A compatible metric $\rho$ on a metrizable $G$-space $X$ is called invariant or $G$ invariant if $\rho(g x, g y)=\rho(x, y)$ for all $g \in G$ and $x, y \in X$. If $\rho$ is a $G$-invariant metric on any $G$-space $X$, then it is easy to verify that the formula

$$
\widetilde{\rho}(G(x), G(y))=\inf \left\{\rho\left(x^{\prime}, y^{\prime}\right) \mid x^{\prime} \in G(x), y^{\prime} \in G(y)\right\}
$$


defines a pseudometric $\widetilde{\rho}$, compatible with the quotient topology of $X / G$. If, in addition, $X$ is a proper $G$-space, then $\widetilde{\rho}$ is, in fact, a compatible metric on $X / G$ 23. Theorem 4.3.4].

For a closed subgroup $H \subset G$, by $G / H$ we will denote the $G$-space of cosets $\{g H \mid g \in G\}$ under the action induced by left translations.

A locally compact group $G$ is called almost connected if the quotient group $G / G_{0}$ of $G$ modulo the connected component $G_{0}$ of the identity is compact.

Such a group has a maximal compact subgroup $K$; i.e., every compact subgroup of $G$ is conjugate to a subgroup of $K$ [1, Theorem A.5]. The corresponding classical theorem on Lie groups can be found in [19, Ch. XV, Theorem 3.1].

In 1961 Palais [23] introduced the very important concept of a proper action of an arbitrary locally compact group $G$ and extended a substantial part of the theory of compact Lie transformation groups to noncompact ones.

Let $X$ be a $G$-space. Two subsets $U$ and $V$ in $X$ are called thin relative to each other [23, Definition 1.1.1] if the set $\langle U, V\rangle=\{g \in G \mid g U \cap V \neq \emptyset\}$, called the transporter from $U$ to $V$, has a compact closure in $G$. A subset $U$ of a $G$-space $X$ is called $G$-small, or just small, if every point in $X$ has a neighborhood thin relative to $U$. A $G$-space $X$ is called proper (in the sense of Palais) if every point in $X$ has a small neighborhood.

Clearly, if $G$ is compact, then every $G$-space is proper. Furthermore, if $G$ acts properly on a compact space, then $G$ has to be compact as well. If $G$ is discrete and $X$ is locally compact, the notion of a proper action is the same as the classical notion of a properly discontinuous action. When $G=\mathbb{R}$, the additive group of the reals, proper $G$-spaces are precisely the dispersive dynamical systems with regular orbit space (see [13, Ch. IV]).

Important examples of proper $G$-spaces are the coset spaces $G / H$ with $H$ a compact subgroup of a locally compact group $G$. The reader can find other interesting examples in [1], 2], [5], 6] and [20].

In what follows we shall also need the definition of a twisted product $G \times_{K} S$, where $K$ is a closed subgroup of $G$, and $S$ a $K$-space. $G \times_{K} S$ is the orbit space of the $K$-space $G \times S$ on which $K$ acts by the rule: $k(g, s)=\left(g k^{-1}, k s\right)$. Furthermore, there is a natural action of $G$ on $G \times{ }_{K} S$ given by $g^{\prime}[g, s]=\left[g^{\prime} g, s\right]$, where $g^{\prime} \in G$ and $[g, s]$ denotes the $K$-orbit of the point $(g, s)$ in $G \times S$. We shall identify $S$ by means of the $K$-equivariant embedding $s \mapsto[e, s], s \in S$, with the $K$-invariant subset $\{[e, s] \mid s \in S\}$ of $G \times_{K} S$. This $K$-equivariant embedding $S \hookrightarrow G \times \times_{K} S$ induces a homeomorphism of the $K$-orbit space $S / K$ onto the $G$-orbit space $\left(G \times_{K} S\right) / G$ (see [15, Ch. II, Proposition 3.3]).

The twisted products are of particular interest in the theory of transformation groups (see [15, Ch. II, $\S 2]$ ). It turns out that every $G$-space locally is a twisted product. For a more precise formulation we need to recall the following well known notion of a slice (see [23, p. 305]).

Definition 2.1. Let $X$ be a $G$-space and $K$ a closed subgroup of $G$. A $K$-invariant subset $S \subset X$ is called a $K$-kernel if there is a $G$-equivariant map $f: G(S) \rightarrow G / K$, called the slicing map, such that $S=f^{-1}(e K)$. The saturation $G(S)$ is called a tubular set, and the subgroup $K$ will be referred to as the slicing subgroup.

If in addition $G(S)$ is open in $X$, then we shall call $S$ a $K$-slice in $X$.

If $G(S)=X$, then $S$ is called a global $K$-slice of $X$. 
It turns out that each tubular set with a compact slicing subgroup is a twisted product. The tubular neighborhood $G(S)$ is $G$-homeomorphic to the twisted product $G \times_{K} S$; namely, the map $\xi: G \times_{K} S \rightarrow G(S)$ defined by $\xi([g, s])=g s$ is a $G$-homeomorphism (see [15, Ch. II, Theorem 4.2]). In what follows we will use this fact without a specific reference.

One of the most powerful results in the theory of topological transformation groups is Palais' slice theorem [23, Proposition 2.3.1], which states that if $X$ is a proper $G$-space with $G$ a Lie group, then for any point $x \in X$, there exists a $G_{x^{-}}$ slice $S$ in $X$ such that $x \in S$. In general, when $G$ is not a Lie group, it is no longer true that a $G_{x}$-slice exists at each point of $X$ (see [4]). Generalizing the case of Lie group actions, in [2] and [7] (see also [4] for the case of compact non-Lie group actions), approximate versions of Palais' slice theorem for non-Lie group actions were proved. Below, in the proof of Theorem 1.12, we shall need the following global slice theorem established by H. Abels [1, Main Theorem]:

Theorem 2.2 (Global Slice Theorem). Let $G$ be an almost connected group, $K$ a maximal compact subgroup of $G$, and $X$ a proper $G$-space with a paracompact orbit space. Then $X$ admits a global $K$-slice.

On any group $G$ one can define two natural (but equivalent) actions of $G$ given by the formulas

$$
g \cdot x=g x \quad \text { and } \quad g * x=x g^{-1},
$$

respectively, where in the right-hand sides the group operations are used with $g, x \in$ $G$.

Throughout we shall consider the second action only.

By $U(G)$ we shall denote the Banach space of all right uniformly continuous bounded functions $f: G \rightarrow \mathbb{R}$ endowed with the supremum norm. Recall that $f$ is called right uniformly continuous if for every $\varepsilon>0$ there exists a neighborhood $O$ of the unity in $G$ such that $|f(y)-f(x)|<\varepsilon$ whenever $y x^{-1} \in O$.

We shall consider the induced action of $G$ on $U(G)$, i.e.,

$$
(g f)(x)=f(x g), \quad \text { for all } g, x \in G .
$$

It is easy to check that this action is continuous, linear and isometric (see, e.g., 3 , Proposition 7]).

Proposition 2.3. Let $G$ be a group. Then for every $f \in U(G)$, the map

$$
f_{*}: G \rightarrow U(G)
$$

defined by $f_{*}(x)(g)=f\left(x g^{-1}\right), x, g \in G$, is a right uniformly continuous $G$-map.

Proof. A simple verification.

\section{Proofs}

Proof of Theorem 1.1. Choose a neighborhood $U$ of the identity in $X$ such that $U=U^{-1}$ and $U^{3} \cap G$ has a compact closure in $G$. We claim that for every $x \in X$, the neighborhood $x U$ is $G$-small. Indeed, let $y \in X$ be any point. Two cases are possible.

Case 1. Assume that $y \in x U^{2} G$. Then $y=x u_{1} u_{2} h$ with $u_{1}, u_{2} \in U$ and $h \in G$. We claim that $x U^{2} h$ is a neighborhood of $y$ thin relative to $x U$. Indeed, if $g \in$ $\left\langle x U, x U^{2} h\right\rangle$, then $g^{-1} h^{-1} \in U^{3} \cap G$. Since $U^{3} \cap G$ has a compact closure, we see 
that so does the set $\left(U^{3} \cap G\right) h$ which contains $g^{-1}$. This yields that $\left\langle x U, x U^{2} h\right\rangle$ is contained in $h^{-1}\left(U^{3} \cap G\right)^{-1}$, which also has a compact closure. Hence the transporter $\left\langle x U, x U^{2} h\right\rangle$ has a compact closure, as required.

Case 2. Assume that $y \notin x U^{2} G$. In this case $y \notin \overline{x U G}$. Indeed, if $y \in \overline{x U G}$, then the neighborhood $x U x^{-1} y$ of $y$ should meet the set $x U G$. Then $x u x^{-1} y=x v h$ for suitable elements $u, v \in U$ and $h \in G$. Then $y=x u^{-1} v h \in x U^{2} G$, a contradiction.

Hence the open set $V=X \backslash \overline{x U G}$ is a $G$-invariant neighborhood of $y$, and $V$ is thin relative to $x U$ because the transporter $\langle x U, V\rangle$ is empty in this case.

Proposition 3.1. Let $X$ be a group and $G$ a locally compact subgroup of $X$. Then there exists a locally finite covering of $X$ consisting of $G$-invariant open sets of the form $S_{i} G$, where each $S_{i}$ is an open $G$-small subset of $X$.

Proof. By Theorem 1.1, $X$ is a proper $G$-space, and hence, one can choose a $G$ small neighborhood $U$ of the unity in $X$. By virtue of Markov's theorem [12, Theorem 3.3.9], there exists a right uniformly continuous function $f: X \rightarrow[0,1]$ such that

$$
f(e)=0 \quad \text { and } \quad f^{-1}([0,1)) \subset U .
$$

Then, by Proposition 2.3, $f$ induces an $X$-equivariant map $f_{*}: X \rightarrow U(X)$ defined by the rule:

$$
f_{*}(x)(g)=f\left(x g^{-1}\right), \quad x, g \in X .
$$

Denote by $Z$ the image $f_{*}(X)$. Clearly, $Z$ is the $X$-orbit of the point $f_{*}(e)$ in the $X$-space $U(X)$, and the metric of $U(X)$ induces an $X$-invariant metric on $Z$. We claim that

$$
f_{*}^{-1}\left(\Gamma_{x, V}\right) \subset x^{-1} U, \quad \text { for every } \quad x \in X,
$$

where $V=[0,1)$ and $\Gamma_{x, V}$ is the open subset $\{\varphi \in U(X) \mid \varphi(x) \in V\}$ of $U$.

First we observe that $\Gamma_{x, V}=x^{-1} \Gamma_{e, V}$ and

$$
f_{*}^{-1}\left(\Gamma_{e, V}\right) \subset f^{-1}(V) .
$$

Then (3.2) follows from (3.1) and the $X$-equivariance of $f_{*}$.

Besides, since $f_{*}(x) \in \Gamma_{x, V}$ for every $x \in X$, we see that the sets $\Gamma_{x, V}, x \in X$, constitute a covering of $Z$.

From now on we restrict ourselves only by the induced actions of the subgroup $G \subset X$; i.e., we will consider $X$ and $Z$ just as $G$-spaces.

Now, it follows from (3.2) and from the $G$-equivariance of $f_{*}$ that

$$
f_{*}^{-1}\left(G\left(\Gamma_{x, V}\right)\right) \subset x^{-1} U G, \quad \text { for every } \quad x \in X .
$$

Since $f_{*}: X \rightarrow Z$ is $G$-equivariant, it induces a continuous map $\widetilde{f}_{*}$ of the $G$-orbit spaces; i.e., we have the following commutative diagram:

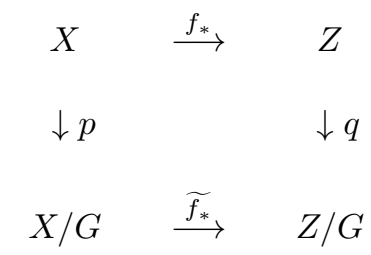

where $p$ and $q$ are the $G$-orbit maps. 
It follows from (3.3) that

$$
\widetilde{f}_{*}^{-1}\left(q\left(\Gamma_{x, V}\right)\right) \subset p\left(x^{-1} U\right), \quad \text { for every } \quad x \in X .
$$

Thus, the open covering $\{p(x U) \mid x \in X\}$ of the $G$-orbit space $X / G$ is refined by the open covering $\left\{\widetilde{f}_{*}^{-1}\left(q\left(\Gamma_{x, V}\right)\right) \mid x \in X\right\}$.

Since the metric of $Z$ is $G$-invariant, the orbit space $Z / G$ is pseudometrizable (see Preliminaries). Hence the open covering $\left\{q\left(\Gamma_{x, V}\right) \mid x \in X\right\}$ of $Z / G$ admits an open locally finite refinement, say $\left\{W_{i} \mid i \in \mathcal{I}\right\}$ (see [16, Theorem 4.4.1 and Remark 4.4.2]). Then, clearly, $\left\{p^{-1}\left(\widetilde{f}_{*}^{-1}\left(W_{i}\right)\right) \mid i \in \mathcal{I}\right\}$ is an open locally finite refinement of $\{x U G \mid x \in X\}$ consisting of $G$-invariant sets. It then follows that each set $p^{-1}\left(\widetilde{f}_{*}^{-1}\left(W_{i}\right)\right)$ is contained in some set $x U G, x \in X$, which yields that

$$
p^{-1}\left(\widetilde{f}_{*}^{-1}\left(W_{i}\right)\right)=S_{i} G,
$$

where $S_{i}=p^{-1}\left(\widetilde{f}_{*}^{-1}\left(W_{i}\right)\right) \cap x U$.

Now, $S_{i}$, being a subset of the $G$-small set $x U$, is itself $G$-small. Thus $\left\{S_{i} G \mid i \in\right.$ $\mathcal{I}\}$ is the desired covering.

Proof of Theorem 1.2. Let $\left\{S_{i} G \mid i \in \mathcal{I}\right\}$ be the locally finite open covering of $X$ from Proposition 3.1. Then the union $S=\bigcup_{i \in \mathcal{I}} S_{i}$ is a $G$-small set (see, e.g., 2, Proposition 1.2(d)]). On the other hand,

$$
S G=\left(\bigcup_{i \in \mathcal{I}} S_{i}\right) G=\bigcup_{i \in \mathcal{I}} S_{i} G=X
$$

yielding that $S$ is a $G$-fundamental subset of $X$. Since the closure of a $G$-small set is $G$-small (see, e.g., [2, Proposition 1.2(b)]), the closure $\bar{S}$ is the desired closed $G$-fundamental set.

Proof of Corollary 1.6. Since $G$ is almost connected, it has a maximal compact subgroup $K$ (see Preliminaries). Since by Corollary 1.5 the quotient $X / G$ is paracompact, due to a result of Abels [1, Main Theorem], $X$ admits a global $K$-slice $S$, and hence it is $G$-homeomorphic to the twisted product $G \times_{K} S$ (see Preliminaries). Since the group $K$ is compact, it then follows that the $K$-orbit map $G \times S \rightarrow G \times_{K} S \cong_{G} X$ is open and perfect. This yields immediately that the restriction $\left.p\right|_{S}: S \rightarrow X / G$ of the $G$-orbit map $p: X \rightarrow X / G$ is an open and perfect surjection. Now the result follows.

Proof of Corollary 1.10. By virtue of Corollary 1.3. $X$ admits a closed subset $F \subset$ $X$ such that the restriction of the quotient projection $p: X \rightarrow X / G$ to $F$ is a perfect surjection $\left.p\right|_{F}: F \rightarrow X / G$. It then follows from the hypothesis that $F$ has the property $\mathcal{P}$. Since a locally compact group is paracompact (even, strongly paracompact [12, Theorem 3.1.1]), then again by the hypothesis, the product $G \times F$ has the property $\mathcal{P}$. Since $F$ is a closed $G$-small set, the action map $G \times F \rightarrow X$ is perfect (see Abels [2, Proposition 1.4]), and since $F G=X$ we see that the map $G \times F \rightarrow X$ is surjective. Then $X$ has the property $\mathcal{P}$ by the hypothesis.

Proof of Corollary 1.11. It is quite similar to the proof of Corollary 1.6. 
Proof of Theorem 1.12. Since $G$ is almost connected, it has a maximal compact subgroup, say, $K$ (see Preliminaries). Since, by Corollary 1.5, the quotient $X / G$ is paracompact, one can apply the Global Slice Theorem (see Theorem 2.2), according to which $X$ admits a global $K$-slice, say $S$. Then $X$ is $G$-homeomorphic to the twisted product $G \times_{K} S$ (see Preliminaries). In turn, due to a result of H. Abels [1, Theorem 2.1], the twisted product $G \times{ }_{K} S$ is homeomorphic to the product $G / K \times S$. Thus, $X \cong G / K \times S$.

Since $G$ is locally compact and paracompact (see [12, Theorem 3.1.1]) and the quotient map $G \rightarrow G / K$ is open and closed, we infer that $G / K$ is also locally compact and paracompact. Further, since $S$ is paracompact, according to a theorem of Morita 21] one has:

$$
\operatorname{dim}(G / K \times S) \leq \operatorname{dim} G / K+\operatorname{dim} S .
$$

Since $K$ is compact, according to a result of V. V. Filippov [17 one has the inequality:

$$
\operatorname{dim} S \leq \operatorname{dim} S / K+\operatorname{dim} q,
$$

where $q: S \rightarrow S / K$ is the $K$-orbit projection and

$$
\operatorname{dim} q=\sup \left\{\operatorname{dim} g^{-1}(a) \mid a \in S / K\right\} .
$$

Further, since $K$ acts freely on $S$, we see that $\operatorname{dim} q=\operatorname{dim} K$.

Consequently, combining (3.5) and (3.6) one obtains:

$$
\operatorname{dim}(G / K \times S) \leq \operatorname{dim} G / K+\operatorname{dim} K+\operatorname{dim} S / K .
$$

Next, since $X / G \cong\left(G \times_{K} S\right) / G \cong S / K$ (see Preliminaries) and $\operatorname{dim} G / K+$ $\operatorname{dim} K=\operatorname{dim} G$ (see Remark 1.13), it then follows from (3.7) that

$$
\operatorname{dim}(G / K \times S) \leq \operatorname{dim} X / G+\operatorname{dim} G,
$$

as required.

\section{ACKNOWLEDGEMENT}

The author thanks the referee for useful comments.

\section{REFERENCES}

1. H. Abels, Parallelizability of proper actions, global $K$-slices and maximal compact subgroups, Math. Ann. 212 (1974), 1-19. MR0375264 (51:11460)

2. H. Abels, A universal proper G-space, Math. Z. 159 (1978), 143-158. MR0501039 (58:18504)

3. S. A. Antonian, Equivariant embeddings into $G$-AR's, Glasnik Matematički 22 (42) (1987), 503-533. MR957632 (89k:54041)

4. S. A. Antonyan, Existence of a slice for arbitrary compact transformation groups, Matematicheskie Zametki 56:5 (1994), 3-9: English transl. in: Math. Notes 56 (5-6) (1994), 101-1104. MR:1330386 (96f:57034)

5. S. A. Antonyan, The Banach-Mazur compacta are absolute retracts, Bull. Acad. Polon. Sci. Ser. Math. 46, no. 2 (1998), 113-119. MR.1631246 (99d:54020)

6. S. A. Antonyan, Extensorial properties of orbit spaces of proper group actions, Topology Appl. 98 (1999), 35-46. MR.1719992

7. S. A. Antonyan, Orbit spaces and unions of equivariant absolute neighborhood extensors, Topology Appl. 146-147 (2005), 289-315. MR2107153(2005i:54022)

8. S. A. Antonyan and S. de Neymet, Invariant pseudometrics on Palais proper G-spaces, Acta Math. Hung. 98 (1-2) (2003), 41-51. MR.1958466 (2003m:22025)

9. A. V. Arhangel'skii, Classes of topological groups, Russian Math. Surveys 36, no. 3 (1981), 151-174. MR622722 (83d:54082) 
10. A. V. Arhangel'skii, Quotients with respect to locally compact subgroups, Houston J. Math. 31, no. 1 (2005), 215-226. MR2123011 (2006a:54028)

11. A. V. Arhangel'skii and V. V Uspenskij, Topological groups: local versus global, Applied General Topology 7, no. 1 (2006), 67-72. MR2284936 (2007k:54042)

12. A. Arhangel'skii and M. Tkachenko, Topological Groups and Related Structures, Atlantis Press/World Scientific, Amsterdam-Paris, 2008. MR2433295

13. N. P. Bhatia, G. P. Szegö, Stability Theory of Dynamical Systems, Springer Verlag, Berlin, 1970. MR0289890 (44:7077)

14. C. J. R. Borges, On stratifiable spaces, Pacific J. Math. 17, no. 1 (1966), 1-16. MR0188982 $(32: 6409)$

15. G. Bredon, Introduction to compact transformation groups, Academic Press, 1972. MR0413144(54:1265)

16. R. Engelking, General Topology, PWN-Pol. Sci. Publ., Warsaw, 1977. MR0500780 $(58: 18316 \mathrm{~b})$

17. V. V. Filippov, Dimensionality of spaces with the action of a bicompact group, Math. Notes, 25, no. 3 (1979), 171-174. MR.534089 (80f:54031)

18. O. Hájek, Parallelizability revisited, Proc. Amer. Math. Soc., 27, no. 1 (1971), 77-84. MR 0271925 (42:6806)

19. G. Hochschild, The structure of Lie groups, Holden-Day Inc., San Francisco, 1965. MR0207883 (34:7696)

20. J. L. Koszul, Lectures on groups of transformations, Tata Institute of Fundamental Research, Bombay, 1965. MR0218485 (36:1571)

21. K. Morita, On the dimension of product spaces, American Journal of Mathematics, 75, no. 2 (1953), pp. 205-223. MR0054240 (14:893c)

22. R. Palais, The classification of $G$-spaces, Memoirs of the AMS, 36 (1960). MR0177401 $(31: 1664)$

23. R. Palais, On the existence of slices for actions of non-compact Lie groups, Ann. of Math. (2) 73 (1961), 295-323. MR0126506 (23:A3802)

24. E. G. Skljarenko, On the topological structure of locally bicompact groups and their quotient spaces, Amer. Math. Soc. Transl., Ser. 2, 39 (1964), 57-82. MR0147575 (26:5090)

Departamento de Matemáticas, Facultad de Ciencias, Universidad Nacional Autónoma de México, 04510 México Distrito Federal, México

E-mail address: antonyan@unam.mx 